EPJ Web of Conferences 35, 03002 (2012)

DOI: $10.1051 /$ epjconf/20123503002

(C) Owned by the authors, published by EDP Sciences, 2012

\title{
Ion-implantation and analysis for doped silicon slot waveguides
}

\author{
L. Deam ${ }^{\mathrm{a}}$, N. Stavrias, K. K. Lee, and J. C. McCallum \\ Micro-analytical Research Centre (MARC), University of Melbourne, Victoria 3010, Australia
}

\begin{abstract}
We have utilised ion implantation to fabricate silicon nanocrystal sensitised erbium-doped slot waveguide structures in a $\mathrm{Si} / \mathrm{SiO}_{2} / \mathrm{Si}$ layered configuration and photoluminescence (PL) and Rutherford backscattering spectrometry (RBS) to analyse these structures. Slot waveguide structures in which light is confined to a nanometre-scale low-index region between two high-index regions potentially offer significant advantages for realisation of electrically-pumped Si devices with optical gain and possibly quantum optical devices. We are currently investigating an alternative pathway in which high quality thermal oxides are grown on silicon and ion implantation is used to introduce the $\mathrm{Er}$ and $\mathrm{Si}$-ncs into the $\mathrm{SiO}_{2}$ layer. This approach provides considerable control over the Er and Si-nc concentrations and depth profiles which is important for exploring the available parameter space and developing optimised structures. RBS is well-suited to compositional analysis of these layered structures. To improve the depth sensitivity we have used a $1 \mathrm{MeV} \alpha$ beam and results indicate that a layered silicon-Er: $\mathrm{SiO}_{2} /$ silicon structure has been fabricated as desired. In this paper structural results will be compared to Er photoluminescence profiles for samples processed under a range of conditions.
\end{abstract}

\section{Introduction}

Current optical interconnects and fibre optic cables operate either between separate computers or over longer distances. Using silicon slot waveguides, these interconnects could be scaled down to function between processors or even within individual chips, permitting further development of optical computing technologies $[1,2]$. In recent years, development of photonic structures using erbiumdoped silicon dioxide layers has generated great interest $[3,4]$.

Slot waveguides were proposed in 2004 by Almeida et al [5]. These structures consist of a thin slot of low refractive index material inside a material of higher refractive index. Various materials have been proposed [6], however a silicon dioxide slot within silicon has many benefits over other materials. In particular, silicon is readily available and its processing is well understood due to its decades of use in the electronics industry.

A useful silicon waveguide would be compatible with current fibre optic technologies. These guide light at the silica conduction band minima, $1.54 \mu \mathrm{m}$, which also corresponds to the ${ }^{4} I_{13 / 2} \rightarrow{ }^{4} I_{15 / 2}$ transition in erbium. Introducing erbium into the slot through ion implantation then allows for optical gain and transmission at $1.54 \mu \mathrm{m}$ along the slot itself. However, exciting erbium ions directly is unlikely as erbium has a very low excitation cross-section, of order $10^{-20} \mathrm{~cm}^{2}$.

Low-dimension silicon structures such as porous silicon or silicon nanocrystals (Si-ncs) have previously been shown to act as sensitisers [7], increasing the erbium excitation cross-section to as high as $10^{-15} \mathrm{~cm}^{2}$ [8]. Figure 1 depicts the transfer and emission process of energy through nanocrystals to erbium.

Various studies have then been focussed on optimising both the formation of silicon nanocrystals and optimis-

\footnotetext{
a e-mail: 1.deam@pgrad.unimelb.edu.au
}

ing conditions for the coupling of erbium to the nanocrystals [9-11]. The implanted silicon and erbium fluence and associated annealling conditions are of particular interest. This study uses ion implantation in both the fabrication and analysis of erbium-doped slot waveguides, including anneals at different temperatures to investigate the evolution of erbium activation. Photoluminescence (PL) and Rutherford backscattering spectrometry (RBS) data allow for complete luminescent and architectural analysis of these structures.

\section{Experimental Techniques}

To create the test waveguide structures, a 4-inch $\langle 100\rangle$ silicon wafer was divided into $\sim 2 \mathrm{~cm}^{2}$ pieces. Samples were cleaned in acetone, deionised water and isopropanol using an ultrasonic bath before stripping of the native oxide using dilute $(4.9 \%)$ hydrofluoric acid. A higher quality 35 nm oxide layer was grown by annealling in a flowing highpurity oxygen ambient at $850^{\circ} \mathrm{C}$ for two hours. After two hours the oxygen was changed to nitrogen for 25 minutes to stop further oxide growth.

Ion implantation was performed on the low energy ion implanter at the Australian National University. Two types of samples were implanted. One sample was implanted with with $10 \mathrm{keV} \mathrm{Si}$ ions at a fluence of $7 \times 10^{15} \mathrm{ions} / \mathrm{cm}^{2}$ followed by an anneal for $1 \mathrm{hr}$ at $1050^{\circ} \mathrm{C}$ in argon to form nanocrystals. Erbium was then implanted at $1.2 \times 10^{14}$ ions $/ \mathrm{cm}^{2}$, followed by an anneal to activate the erbium, as detailed later in this section. Both species were implanted at 30 degrees to the surface normal to reduce the implantation depth and to give a more uniform implantation profile across the slot layer. The second sample had no silicon implanted or associated anneal, so no nanocrystal formation anneal, and was implanted with erbium to the same fluence as the first sample. To summarise the annealling process; in keeping with the literature, for samples implanted with 


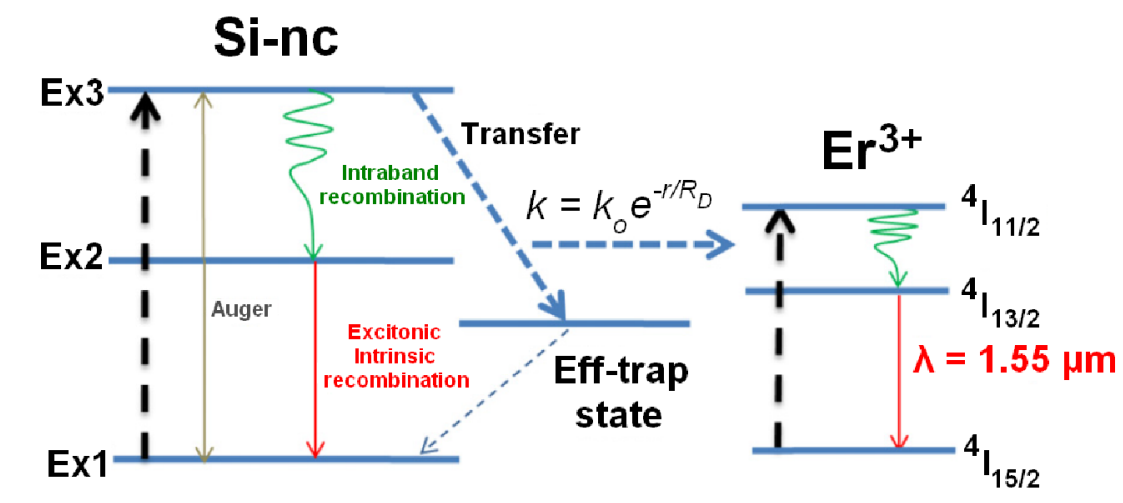

Fig. 1. Excitation and energy transfer mechanism from silicon nanocrystals to $\mathrm{Er}^{3+}$ ions. After [13].

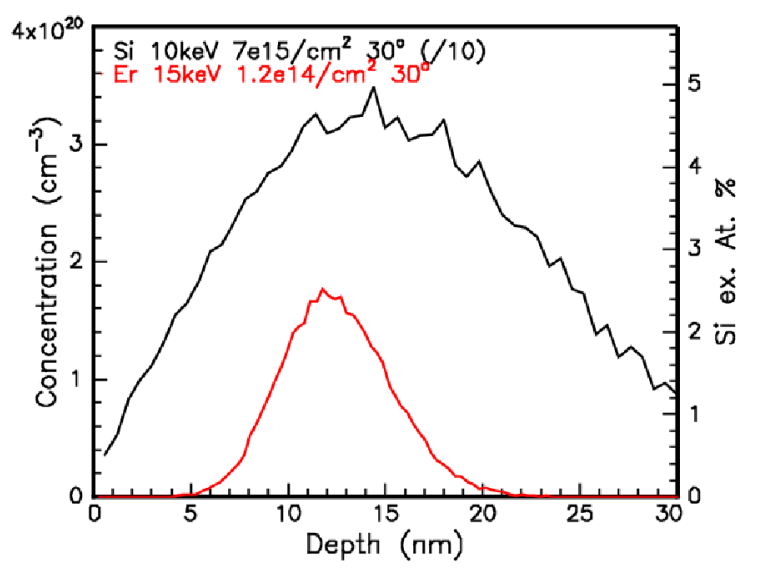

Fig. 2. SRIM simulation of silicon (black) and erbium (red) implanted into a $\sim 35 \mathrm{~nm} \mathrm{SiO} 2$ layer.

excess silicon and erbium, a two-stage annealling process is performed, first to form nanocrystals at $1050^{\circ} \mathrm{C}$ and secondly to activate the erbium with an anneal in the range $700-900^{\circ} \mathrm{C}$, detailed later in this section.

Figure 2 shows SRIM [12] simulations of the implanted silicon and erbium, predicting a peak silicon concentration of 5 atomic.\% excess at a depth of $20 \mathrm{~nm}$. Atomic percentage excesses below $10 \%$ are considered 'dilute' systems [7]. In this regime, the average size of nanocrystals formed is constant near $\sim 3 \mathrm{~nm}$, with increasing silicon fluence only increasing nanocrystal concentration. Previous work has looked at silicon concentrations above this limit and observed nanocrystals using similar processing conditions to ours [14].

After implantation the wafer pieces were divided in half. One half had $150 \mathrm{~nm}$ of amorphous silicon deposited on the surface, creating a complete slot waveguide structure. The other half was left without a capping layer to investigate solely the coupling of erbium to nanocrystals without any waveguiding behaviour.

After samples had been implanted, divided and had a capping layer deposited, each of the samples was further divided into four to allow for different thermal treatments. One section was left as-implanted, with no thermal treatment. To investigate optical activation of the erbium, the other samples were annealled in flowing nitrogen for $1 \mathrm{hr}$ at temperatures of either 700,900 or $1100^{\circ} \mathrm{C}$. Note that this anneal is independent of the anneal required to form nanocrystals; a two-stage process has been performed to independently form nanocrystals and subsequently activate the erbium. Limiting any oxide growth during this second anneal is desirable as oxide growth would either shift the Er-doped oxide away from the silicon substrate in uncapped samples or would reduce the silicon cap layer thickness in capped samples. Oxygen levels reduced by passing the nitrogen gas through oxygen traps and a coil immersed in liquid nitrogen, which condenses any remaining oxygen.

To summarise the fabricated samples; two wafer pieces were initially implanted, one with $\mathrm{Si}$ and $\mathrm{Er}$, the other with only Er. Both samples were divided in half, one half having a silicon cap deposited. Each of these four samples were then divided into four to allow for different thermal treatments. Samples deposited with a silicon capping layer will be referred to as "capped" in text or "w cap" in figures.

Photoluminescence measurements were performed using a $532 \mathrm{~nm}$ laser on a Renishaw micro Raman spectroscopy system. Nanocrystals show up as a broad band in the region $600-900 \mathrm{~nm}$ (in the visible range), while erbium has a sharp peak centered on $1535 \mathrm{~nm}(\sim 1.54 \mu \mathrm{m})$ in the near infra-red. The visible range measurements were taken using a $1200 \mathrm{~g} / \mathrm{mm}$ grating and a charge-coupled device (CCD), while the near infra-red used a $830 \mathrm{~g} / \mathrm{mm}$ grating and a liquid nitrogen cooled InGaAs array detector.

Rutherford Backscattering Spectrometry (RBS) measurements were taken using the $5 \mathrm{MV}$ NEC pelletron accelerator at the University of Melbourne. A beam of $1.1 \mathrm{MeV}$ $\alpha$ particles was used, with a backscatter detector located at $10^{\circ}$ to the sample surface normal. The average charge collected for each sample was $5 \mu C$.

\section{Results and discussion}

\subsection{Photoluminesence measurements}

Figure 3 shows PL spectra for samples annealled at $700^{\circ} \mathrm{C}$. The peak centred around $1535 \mathrm{~nm}$ is the erbium transition; the secondary peak near $1555 \mathrm{~nm}$ is due to Stark splitting. As seen in this figure, the strongest signal comes from the capped, Er-only sample. That the cap layer enhances the signal is expected as the cap serves to confine light within the slot, and also introduces defects at the $\mathrm{SiO}_{2} / \mathrm{Si}$ cap layer interface that may act as sensitisers.

Noticeably, the capped sample implanted with both $\mathrm{Si}$ and Er did not produce an erbium signal. The same was 


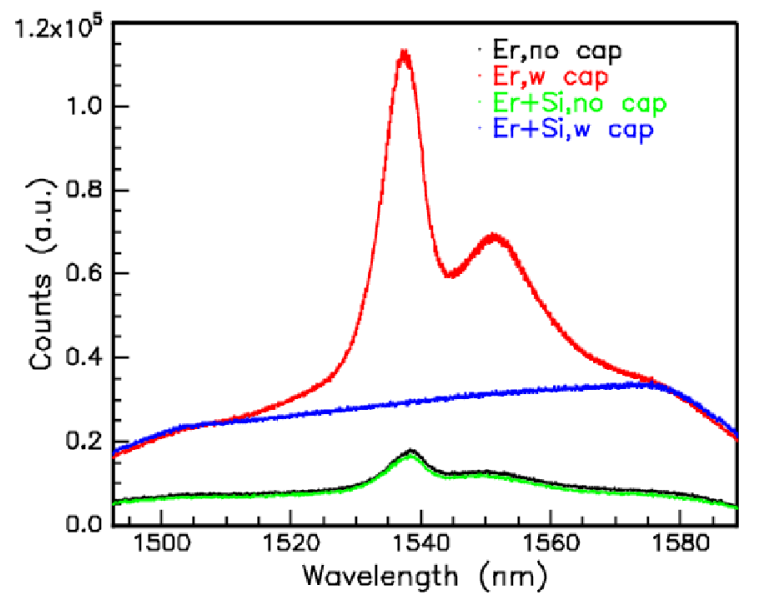

Fig. 3. Photoluminescence spectra of samples after annealling at $700^{\circ} \mathrm{C}$. Note that " $\mathrm{Er}+\mathrm{Si}$ w cap" shows no erbium luminescence.

found for the other capped Si- and Er-implanted samples, regardless of annealling temperature. Initially it was considered that the Si-implanted/capped combination may be causing some kind of quenching effect, however RBS results (figure 6) revealed there to be no erbium in this particular section of the sample.

Figure 3 also shows identical PL spectra for both uncapped samples. This similarity was observed across all uncapped samples, as plotted in figure 4, suggesting that there are no or very few nanocrystals in any of the Siimplanted samples. Separate PL measurements were taken in the nanocrystal luminescence range, however no signal was observed. Various factors could be responsible for this lack of nanocrystals, in particular the annealling ambient and implanted silicon concentration. These are elaborated on in section 3.3.

Figure 4 shows the trend of the erbium luminescence with annealling temperature for samples that produced an Er signal. Of these, the same trend is observed for all samples, with the largest signal after a $700^{\circ} \mathrm{C}$ anneal, and a decrease in signal intensity above this. Reports of a large drop in signal for anneals above $1000^{\circ} \mathrm{C}$ are common [15, 16]. The ideal annealling temperature also shifts with implanted silicon fluence and subsequent nanocrystal formation [15], meaning this result may change with correctly formed nanocrystals.

It would seem then that several questions were raised from these PL spectra, however we are also interested in the architecture of the samples themselves. Did they match our expectations from our processing conditions, or are there changes that need to be made?

\subsection{Rutherford backscattering spectrometry measurements}

Figures 5 and 6 show RBS profiles of two samples with overlaid RUMP [17] simulations in red.

Figure 5 shows a sample with a silicon cap layer but without any thermal treatment. As indicated, this spectra shows a silicon cap layer on top of an Er-doped $\mathrm{SiO}_{2}$ slot layer. Simulations confirm that there is both an oxide slot layer below the silicon cap, corresponding to the dip in

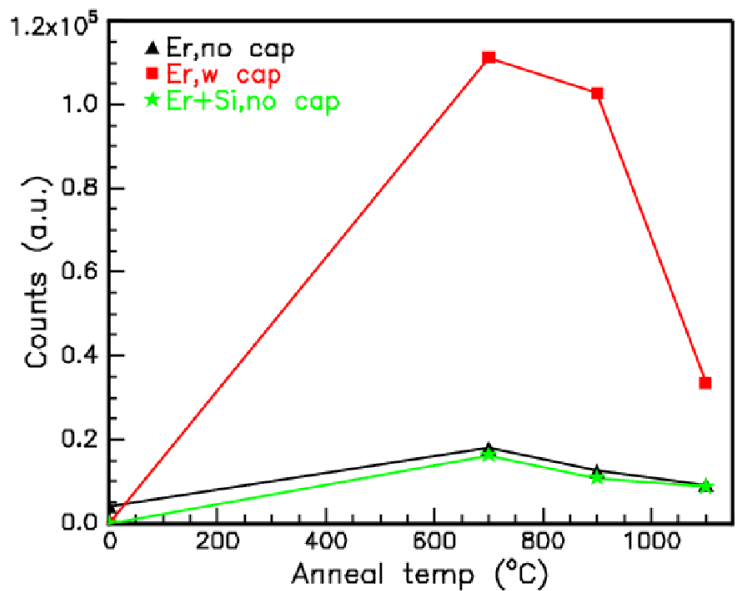

Fig. 4. Erbium PL luminescence for samples annealled at different temperatures.

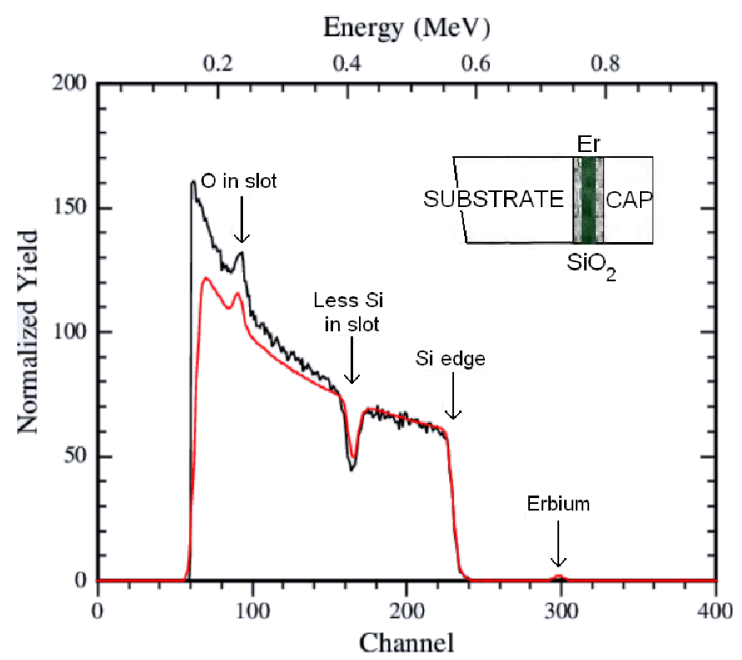

Fig. 5. Sample with capping layer but no thermal treatment.

signal and a corresponding oxygen peak, and that erbium is correctly placed inside this slot.

Also important to note are the lack of any significant surface oxide, less than $\sim 5 \mathrm{~nm}$ here, indicating that the silicon cap deposition did not induce oxide growth. The RUMP simulation for this sample allows for both the slot and capping layer thicknesses to be determined, in this case $\sim 300 \mathrm{~nm}$ and $\sim 35 \mathrm{~nm}$ respectively. Similar numbers were obtained for other samples. This cap layer thickness is then roughly double what was intended to be deposited, suggesting the deposition time may be an overestimate.

Figure 6 shows an RBS spectrum for an Er- and Siimplanted capped sample annealled at $1100^{\circ} \mathrm{C}$. While the RUMP simulation predicts erbium near channel 295, no such signal is seen in the experimental spectra. As noted in section 3.1, none of the capped Er- and Si-implanted samples produced Er luminescence. Rather than any quenching effect, the combined PL and RBS data indicate simply a lack of erbium in the samples analysed. The implanted area was smaller than the sample size, resulting in unimplanted sections being analysed in this case. Upon performing RBS on other sections of this sample, erbium was located. Both PL and RBS data regarding the presence of erbium agree across all samples. 


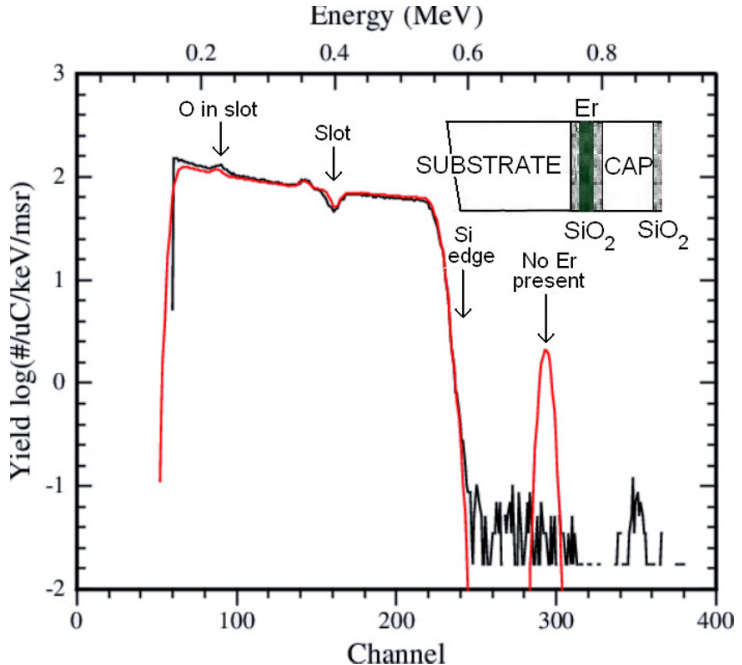

Fig. 6. Sample with capping layer and $1100^{\circ} \mathrm{C}$ anneal. Note the $\log$ scale to better show the lack of erbium.

On comparison with unannealled samples, RUMP simulations predict that a surface oxide of thickness $\sim 20 \mathrm{~nm}$ has grown during the erbium activation anneals. While this surface oxide thickness varied across samples, any samples annealled, with or without a cap layer, had a surface oxide present. Given a slot thickness of $\sim 35 \mathrm{~nm}$, this (lowerquality) oxide acts to almost double the slot thickness in uncapped samples. Subsequent anneals on different samples demonstrated that purging the furnace for longer before annealling produced noticably thinner (few $\mathrm{nm}$ ) oxides.

Table 1 compares the expected architecture and other variables to experimental results.

\subsection{Additional treatments to form nanocrystals}

The lack of nanocrystals across all Si-implanted samples was curious. A variety of factors could have been responsible for the nanocrystals not forming. We were interested in investigating both the annealling ambient used and implanted silicon fluence.

Some extra samples were prepared and have so far produced negative results. Some samples from this initial set were annealled in an argon and hydrogen mixture, rather than argon, to attempt to form nanocrystals. At this point it was considered that the samples may not have enough additional implanted silicon for the excess silicon to agglomerate into nanocrystals. One sample that had already been implanted with $\mathrm{Si}$ and annealled in the $\mathrm{Ar} / \mathrm{H}_{2}$ mixture was implanted again with a 'top up' of silicon at $1 \times$ $10^{16}$ ions $/ \mathrm{cm}^{2}$. This brought the total implanted fluence to $1.7 \times 10^{16}$ ions $/ \mathrm{cm}^{2}$, with the peak concentration of silicon to 11 atomic.\% excess at $20 \mathrm{~nm}$. This is above the 'dilute' system limit. This too did not result in any nanocrystal formation, but could possibly be attributed to damage effects, nanocrystal malformation or structural changes in the slot due to the dual implant and anneal.

Before investigating other parameters one further implantation is planned. This will be a single implant of silicon at $1.7 \times 10^{16}$ ions $/ \mathrm{cm}^{2}$. If no nanocrystal luminescence is observed at this fluence other causes will be investigated.
Table 1. Expected sample composition and experimental result.

\begin{tabular}{lll}
\hline & Design & Experiment \\
\hline Cap thickness & $\sim 150 \mathrm{~nm}$ & $\sim 300 \mathrm{~nm}$ \\
Slot thickness & $\sim 30 \mathrm{~nm}$ & $\sim 35 \mathrm{~nm}$ \\
Nanocrystals formed & Yes & No \\
Erbium implanted & Yes & Yes \\
Oxide growth during anneal & No & Yes, $\sim 20 \mathrm{~nm}$
\end{tabular}

One possibility considered is that there exists a minimum critical slot thickness, below which nanocrystals are unable to form in silicon dioxide.

\section{Conclusions}

Test silicon slot waveguides were created using ion implantation and analysed using photoluminescence measurements and Rutherford backscattering spectrometry. Erbium or silicon and erbium were implanted into $\mathrm{SiO}_{2}$ slot layers to investigate luminescence properties at $1.54 \mu \mathrm{m}$. The implanted silicon was intended to form into nanocrystals to increase emission at this wavelength. Photoluminescence measurements demonstrated silicon nanocrystals were not present in any of the Si-implanted samples. The reasons for this are currently being investigated but likely relate to the implanted silicon fluence being too low to form nanocrystals.

PL measurements on annealled samples supported previous works suggesting anneals beyond $1000^{\circ} \mathrm{C}$ result in a reduction in luminescence at the desired wavelength. Photoluminescence measurements also showed much stronger emission when a silicon capping layer was present than without. RBS measurements confirmed the waveguide structures had been formed, and suggested some changes to processing steps that could be made to more closely match intended designs.

The combination of photoluminescence and Rutherford backscattering spectrometry techniques have been demonstrated to be very useful in the analysis of slot waveguide structures.

Beyond fabrication of these slot waveguides, future work will involve more detailed characterisation of waveguide properties. Given that erbium has a small excitation crosssection, quantum efficiency measurements will be important in ascertaining the long-term viability of completed structures and their integration with existing optical devices. This is a planned characterisation step once more complete waveguide structures have been fabricated.

\section{References}

1. Miller et al., Proceedings of the IEEE 97, No. 7, (2009) 1166-1185

2. Krishnamoorthy et al., Proceedings of the IEEE 97, No. 7, (2009) 1337-1361

3. Liang et al., Nature Photonics 4, (2010) 511-517

4. Galli et al., Applied Physics Letters 89, (2010) 241114

5. Almeida et al., Optics Letters 29, (2004) 1209-1211

6. Hiscocks et al., Optics Express 17, (2009) 7295 
7. Garrido Fernandez et al., Journal of Applied Physics 91, (2001), 798-807

8. Gourbilleau et al., Journal of Applied Physics 95, (2004), 3717

9. Miller et al., Journal of Applied Physics 108, (2010) 063109

10. Franzò et al., Applied Physics Letters 90, (2007), 183102

11. Fujii et al., Applied Physics Letters 71 (9), (1997) $1198-1200$

12. J. Ziegler, The Stopping and Range of Ions in Matter, 2008 http://www.srim.org/

13. Navarro-Urrios et al., Optical Materials 33, (2011) 1086

14. Ryan et al., Masters Thesis (2011), School of Physics, University of Melbourne

15. Hijazi et al., Journal of Applied Physics 106, (2009), 024311

16. Carrada et al., Optical Materials 27, (2005) 915-919

17. M. Thompson, http://www.genplot.com/ 